\title{
La calcolosi molle e le potenzialità del trattamento endourologico
}

\author{
P. Beltrami, L. Ruggera, F. Gigli, A. Aloisi, F. Zattoni \\ Cattedra e Divisione Clinicizzata di Urologia, Università degli Studi di Verona
}

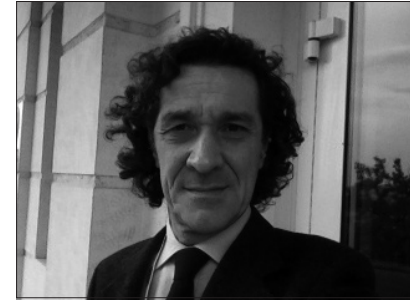

\section{Introduzione}

La calcolosi renale "molle", conosciuta anche con il nome di calcolosi colloide o di albumina e descritta per la prima volta nel 1908 da Gage e Beal (1), rapP. Beltrami

pre- senta una varietà di litiasi urinaria raramente diagnosticata nella pratica clinica e così definita principalmente per la sua caratteristica macroscopica di carenza o assenza di una componente minerale dura.

Rispetto alla litiasi tradizionale, questi calcoli sono di consistenza molle, deformabili, amorfi in quanto la matrice ammonta a circa il $65 \%$ del peso secco rispetto al $2.5 \%$ quale si riscontra generalmente negli altri tipi di calcoli. Alle indagini radiologiche convenzionali, tali calcoli appaiono radiotrasparenti o debolmente radioopachi in quanto la componente minerale risulta essere poco rappresentata (2). Le varie metodiche di imaging non sono in genere dirimenti e spesso non sono in grado di distinguere questi difetti di riempimento da quelli determinati dalla presenza di una neo-plasia. La diagnosi pertanto è più spesso endoscopica, intraoperatoria. In letteratura si è riferito di circa 60 casi, trattati con approccio chirurgico e dal 1990 anche con litotrissia percutanea (3-5).

Riportiamo qui la nostra esperienza di questa relativamente rara forma di litiasi renoureterale, trattata con metodica endourologica.

\section{Pazienti e Metodi}

La nostra esperienza comprende 8 pazienti, di sesso femminile, di età compresa tra 4 e 69 anni (media 44.4 anni). In 4 casi, le pazienti riferivano una qualche sintomatologia dolorosa al fianco; in 2 casi, il riscontro è avvenuto in seguito ad accertamenti per il ripetersi di infezioni urinarie. Negli altri 2 casi, era invece presente una batteriuria asintomatica.

Per quanto riguarda la sede e le caratteristiche dei calcoli, in 3 pazienti il calcolo era localizzato a livello della pelvi renale; in un caso a livello della pelvi e dei calici superiori; due pazienti erano portatrici di una calcolosi a stampo; in un caso il calcolo era localizzato solo nei calici superiori. Infine nel rimanente caso più calici erano occupati da calcoli (Tab. I).

Il trattamento è stato scelto in relazione alla sede e alle dimensioni dei calcoli. In caso di approccio percutaneo, l'accesso al rene è stato attraverso i calici inferiori. I vari sistemi di frammentazione risultano assolutamente inefficaci su questi calcoli e si deve cercare piuttosto di aspirare la componente molle o di asportarla con pinza. Nel caso vi sia associata una componente solida, questa è favorevolmente frammentata con sonda ultrasonica. Nel caso si utilizzi la via transureterale retrograda, si può pensare di ridurre la componente molle, utilizzando il laser ad olmio. L'asportazione della componente molle viene meglio eseguita con pinza o cestello. In taluni casi abbiamo collegato direttamente un aspiratore allo strumento endoscopico. La litotrissia extracorporea (ESWL) è stata impiegata solo nel caso in cui una componente "solida" fosse residuata al trattamento endourologico.

\section{Risultati}

In 7 casi abbiamo ottenuto la bonifica completa con 6 trattamenti di litotrissia percutanea, 2 litotrissie per via ureterorenoscopica e una ESWL. Nessuna paziente ha sviluppato recidive. Un caso viene invece discusso qui di seguito a causa delle numerose recidive e dei diversi 
TABELLA I - PAZIENTI, SEDE DEI CALCOLI, TRATTAMENTO E RISULTATI

\begin{tabular}{|c|c|c|c|c|c|c|c|}
\hline $\mathbf{P z}$ & $\begin{array}{l}\text { Età } \\
\text { (aa.) }\end{array}$ & $\begin{array}{l}\text { Infezione } \\
\text { urinaria }\end{array}$ & Sede & $1^{\circ}$ Trattamento & Risultato & $2^{\circ}$ Trattamento & Risultato \\
\hline 1 & 4 & - & Ampolla & PCNL & $\begin{array}{l}\text { Clearance } \\
\text { completa }\end{array}$ & & \\
\hline 2 & 65 & Presente & Ampolla & PCNL & $\begin{array}{l}\text { Clearance } \\
\text { completa }\end{array}$ & & \\
\hline 3 & 49 & Batteriuria & Ampolla e calici inferiori & PCNL & $\begin{array}{l}\text { Clearance } \\
\text { completa }\end{array}$ & & \\
\hline 4 & 27 & Batteriuria & A stampo completa & PCNL & $\begin{array}{l}\text { Clearance } \\
\text { completa }\end{array}$ & & \\
\hline 5 & 35 & - & A stampo completa & PCNL & $\begin{array}{l}\text { Clearance } \\
\text { completa }\end{array}$ & & \\
\hline 6 & 69 & - & Ampolla & URS & Residui & ESWL & Clearance completa \\
\hline 7 & 56 & - & Calici superiori & URS & Residui & PCNL & Clearance completa \\
\hline 8 & 50 & Presente & Caliceale multipla & (Descrizione a parte) & & & \\
\hline
\end{tabular}

trattamenti utilizzati (Tab. I).

Si è trattato di una paziente affetta da calcolosi multipla caliceale, sottoposta inizialmente a intervento di bonifica della calcolosi per via transureterale retrograda in due circostanze successive. Quindi, per il riformarsi del calcolo è stata eseguita una litotrissia percutanea e una ESWL, sempre ottenendo una completa bonifica della calcolosi presente. Per la comparsa di una ulteriore recidiva, in altra sede, è stata sottoposta a intervento chirurgico, per via aperta, di pielocalicolitotomia. Dopo appena alcune settimane dall'intervento, in presenza di una persistente infezione urinaria sostenuta da Proteus mirabilis, si è verificata una ulteriore recidiva litiasica trattata, in questa occasione, con una litotrissia percutanea. Anche in questo caso, la bonifica è stata completa. Nei mesi successivi, nonostante una adeguata profilassi, che comunque non ha consentito la eradicazione della infezione urinaria, sempre presente, la paziente è andata incontro a nuove recidive calcolotiche ed è stata quindi sottoposta, periodicamente, a trattamenti per via transureterale. Le indagini di imaging hanno documentato la presenza di una dilatazione dei calici inferiori che contenevano dei calcoli, con importante corticalizzazione. Si decideva così di eseguire una resezione polare inferiore. A più di 12 mesi di follow-up, la paziente è tuttora libera da calcoli e non ha più presentato alcuna infezione urinaria.

Nei casi trattati, l'esame chimico-fisico dei calcoli asportati ha evidenziato la presenza di materiale proteico amorfo, per ciò che riguarda la componente molle, e, cir- ca la componente minerale, fosfocarbonato di calcio (in 1 caso), ossalato di calcio monoidrato (1 caso), acido urico e urato monoammonico (2 casi) e fosfato ammoniomagnesiaco (4 casi).

\section{Discussione}

La calcolosi molle è una rara forma di calcolosi che si riscontra infrequentemente nella pratica clinica. In questa varietà di calcolosi la quantità di matrice ammonta mediamente al $65 \%$ del peso secco e la componente minerale risulta essere presente in una ridotta quantità (2). Boyce e Garvey hanno dimostrato che questa sostanza organica è simile, anche se non identica, alla matrice presente nei calcoli "duri" e risulta costituita per un terzo da mucopolisaccaridi acidi e per due terzi da proteine, a loro volta contenenti esosi ed esosamine per quanto riguarda la componente polisaccaridica e gli aminoacidi treonina, leucina, serina, tiroxina e arginina, per la componente proteica (6).

Nonostante la microscopia elettronica abbia evidenziato che i calcoli di matrice in pazienti con proteinuria e in emodialisi abbia una struttura fibrillare, il materiale proteico risulta essere diverso dalla proteina di TammHorsfall (7). II ruolo della matrice proteica nella formazione dei calcoli non è completamente noto: alcuni Autori ritengono che la matrice possa fungere da supporto su cui si depositerebbero i cristalli; secondo altri, la componente proteica potrebbe precipitare spontaneamente 
insieme ai cristalli $(8,9)$. Ignota tuttavia è la causa della ridotta o mancata precipitazione di cristalli, nel caso di una calcolosi "molle". In questa particolare categoria di pazienti la mancata calcificazione della matrice potrebbe essere legata a una ridotta eliminazione di calcio urinario da parte del rene interessato. La normocalciuria riscontrata nella maggior parte dei casi sarebbe determinata da una maggiore escrezione di calcio da parte del rene controlaterale (3).

La calcolosi renale "molle" è stata descritta in pazienti di tutte le età. Colpisce soprattutto il sesso femminile con anamnesi positiva per infezioni urinarie recidivanti soprattutto sostenute da Proteus o Escherichia Coli (5). Nella nostra esperienza, tutti i pazienti erano di sesso femminile; in 2 casi l'anamnesi era positiva per infezioni urinarie recidivanti e in 2 casi era presente batteriuria asintomatica.

La modalità d'esordio e il quadro clinico della calcolosi molle è sovrapponibile a quella dei pazienti affetti da litiasi calcica o uratica, sebbene possa essere più frequentemente responsabile di quadri di insufficienza renale a causa della graduale ostruzione delle cavità pielo-caliceali, che tende a riprodurre a stampo (10).

Le indagini radiologiche convenzionali non sono in grado di evidenziare con sicurezza questo tipo di calcoli in quanto appaiono radiotrasparenti o debolmente radioopachi, per il basso contenuto di minerali (2). Anche la diagnostica di imaging risulta particolarmente difficile per l'assenza di un quadro patognomonico. Tale nefrolitiasi è infatti caratterizzata da un'ampia variabilità di quadri radiologici, dal riscontro di aria all'interno della via escretrice, a sospetti difetti di riempimento prevalentemente localizzati a livello pielo-caliceale. L'urografia non risulta dirimente in merito alla natura di tali difetti "di riempimento", anche in considerazione della possibile riduzione della funzionalità renale riscontrabile in questi casi, con conseguente diminuita eliminazione del mezzo di contrasto. Alla ecografia, la calcolosi molle viene identificata come una struttura solida priva delle caratteristiche specifiche della nefrolitiasi. L'assenza della marcata iperecogenicità e del cono d'ombra posteriore, patognomoniche della calcolosi calcica, sono verosimilmente da attribuire al basso contenuto minerale di questo tipo di litiasi (11). Analogamente, anche la TC non risulta sempre efficace nella diagnosi differenziale della calcolosi "molle" a causa dell'ampia variabilità di quadri radiologici, conseguente alla diversa percentuale minerale presente (12). Alcuni Autori, infine, attribuiscono un possibile ruolo della RM nello studio della calcolosi "molle". Tale metodica caratterizzata dall'utilizzo di mezzi di contrasto non nefrotossici, è in grado di identificare questi calcoli come difetti di riempimento, solita- mente ipointensi nelle immagini T1 pesate e lievemente iperintensi nelle immagini T2 (13).

Nella maggior parte dei casi, pertanto, l'aspetto diagnostico di imaging non appare indicativo di calcolosi "molle" e la diagnosi risulta di necessità endoscopica intraoperatoria.

Due fattori devono essere tenuti in considerazione quando si pone l'indicazione al trattamento di una calcolosi molle: il potenziale rapido accrescimento e una difficoltà intrinseca alla progressione lungo la via escretrice e quindi all'espulsione spontanea. Anche calcoli di piccole dimensioni e asintomatici possono pertanto risultare meritevoli di un trattamento, proprio perché difficilmente vengono eliminati.

La ESWL appare inefficace nel trattamento di questo tipo di nefrolitiasi a causa della ridotta o assente componente minerale del calcolo. La mobilità del calcolo, legata alla sua componente gelatinosa, rappresenta una ulteriore limitazione alla efficacia dell'ESWL. Nella nostra casistica, la litotrissia extracorporea è stata impiegata soltanto per il trattamento di frammenti residui con evidente componente minerale.

Mentre in passato il trattamento di scelta in caso di calcolosi "molle" era rappresentato dall'intervento chirurgico "a cielo aperto", attualmente questo è stato rimpiazzato dall'approccio endourologico, sia per via percutanea che per via retrograda $(3,5)$. Nella nostra esperienza la PCNL si è rivelata la metodica di scelta risultando in grado di portare alla bonifica completa in una unica seduta e senza ulteriori trattamenti.

L'approccio per via retrograda è stato più di recente impiegato nel trattamento della patologia litiasica anche delle cavità intrarenali e trova quindi spazio anche in questo particolare tipo di calcolosi. Nei casi dubbi, dal punto di vista diagnostico, I'URS si rivela indispensabile per escludere una neoplasia della via escretrice (5) e può, di contro, risultare una ottima alternativa terapeutica se le dimensioni della massa litiasica sono contenute.

Al momento, in letteratura, non sono disponibili misure di profilassi atte a prevenire la formazione di questo tipo di calcolosi. La bonifica il più possibile completa e, unitamente, l'eliminazione della infezione urinaria/batteriuria sono elementi fondamentali per ridurre il rischio di recidiva.

\section{Conclusioni}

L'approccio endourologico è attualmente considerato di prima scelta nel trattamento della calcolosi "molle". La PCNL consente, nella maggior parte dei casi, la bonifica 
completa in una unica seduta. L'ureterorenoscopia, da impiegare anche a scopo diagnostico nei casi dubbi, può essere terapeutica se la massa litiasica è di volume limitato. È comunque da tenere in considerazione come opzione di prima scelta nei casi in cui vi siano frequenti recidive e a breve distanza di tempo. L'ESWL va impiegata esclusivamente per il trattamento di eventuali residui ad elevato contenuto minerale essendo completamente inefficace sulla componente molle. Una adeguata profilassi delle infezioni urinarie e della batteriuria asintomatica e uno stretto follow-up, da continuarsi nel tempo, sono indispensabili in questi pazienti.

Indirizzo degli Autori:

Paolo Beltrami, MD

Divisione Clinicizzata di Urologia

Policlinico "G.B. Rossi"

Piazza L.A. Scuro, 10

37134 Verona, Italy

paolo.beltrami@azosp.vr.it

\section{Bibliografia}

1. Gage $\mathrm{H}$ and Beal $\mathrm{H} \mathrm{W}$. Fibrinous calculi in the kidney. Ann Surg 1908; 48(3): 378-87.

2. Allen TD and Spence HM. Matrix stones. J Urol 1966; 95(3): 284-90.

3. Bahni-Hani AH, Segura JW, Leroy AJ. Urinary matrix calculi: our experience at a single institution.
J Urol 1990; 173(1): 120-23.

4. Stoller ML, Gupta M, Bolton D, Irby PB 3rd. Clinical correlates of the gross, radiographic, and histologic features of urinary matrix calculi. J Endourol 1994; 8(5): 335-40.

5. Shah HN, Kharodawala S, Sodha HS, Khandkar AA, Hegde SS, Bansal MB. The management of renal matrix calculi: a single-centre experience over 5 years. BJU Int 2009; 103(6): 810-4.

6. Boyce WH and Garvey FK. The amount and nature of the organic matrix in urinary calculi: a review. J Urol 1956; 76(3): 213-27.

7. Bommer J, Ritz E, Tschöpe W, Waldherr R, Gebhardt M. Urinary matrix calculi consisting of microfibrillar protein in patients on maintenance hemodialysis. Kidney Int 1979; 16(6): 722-8.

8. Boyce WH and King JS Jr. Crystal-matrix interrelations in calculi. J Urol 1959; 81(3): 351-65.

9. Vermeulen CW, Lyon ES, Gill WB. Artificial urinary concretions. Invest Urol 1964; 1: 370-86.

10. Matthews LA, Spirnak JP. A matrix calculus causing bilateral ureteral obstruction and acute renal failure. $J$ Urol 1995; 154(3): 1125-6.

11. Zwirewich CV, Buckley AR, Kidney MR, Sullivan LD, Rowley VA. Renal matrix calculus. Sonographic appearance. J Ultrasound Med 1990; 9(1): 61-4.

12. Kim SH, Lee SE, Park IA. Care Report. CT and US features of renal matrix stones with calcified center. $J$ Comput Assist Tomogr 1996; 20(3): 404-6.

13. Liu CC, Li CC, Shih MC, Chou YH, Huang CH. Matrix stones. J Comput Assist Tomogr 2003; 27(5): 810-3. 